\title{
Erratum: Short-Term Plasticity and Long-Term Potentiation in Magnetic Tunnel Junctions: Towards Volatile Synapses [Phys. Rev. Applied 5, 024012 (2016)]
}

Abhronil Sengupta and Kaushik Roy

(Received 13 July 2017; published 25 July 2017)

DOI: 10.1103/PhysRevApplied.8.019903

A typographical error was introduced in Eq. (2). The correct equation appears below:

$$
\frac{1+\alpha^{2}}{\gamma} \frac{d \hat{\mathbf{m}}}{d t}=-\left(\hat{\mathbf{m}} \times \mathbf{H}_{\mathrm{eff}}\right)-\alpha\left(\hat{\mathbf{m}} \times \hat{\mathbf{m}} \times \mathbf{H}_{\mathrm{eff}}\right)+\frac{1}{q \gamma N_{s}}\left[\alpha\left(\hat{\mathbf{m}} \times \mathbf{I}_{s}\right)-\left(\hat{\mathbf{m}} \times \hat{\mathbf{m}} \times \mathbf{I}_{s}\right)\right] .
$$

This does not affect any other equations, results, or conclusions reported in the paper. 